\title{
The RNFT2/IL-3R $\alpha$ axis regulates IL-3 signaling and innate immunity
}

\author{
Yao Tong, ${ }^{1,2}$ Travis B. Lear, ${ }^{1,3,4}$ John Evankovich, ${ }^{1,4}$ Yanwen Chen, ${ }^{4,5}$ James D. Londino, ${ }^{1,6}$ \\ Michael M. Myerburg, ${ }^{1}$ Yingze Zhang, ${ }^{1}$ Iulia D. Popescu, ${ }^{1}$ John F. McDyer, ${ }^{1}$ Bryan J. McVerry, ${ }^{1,3}$ \\ Karina C. Lockwood, ${ }^{4}$ Michael J. Jurczak, ${ }^{7}$ Yuan Liu, ${ }^{1,4,8}$ and Bill B. Chen ${ }^{1,4,9}$ \\ ${ }^{1}$ Acute Lung Injury Center of Excellence, Division of Pulmonary, Allergy and Critical Care Medicine, Department of \\ Medicine, School of Medicine, University of Pittsburgh, Pittsburgh, Pennsylvania, USA. ²Department of Anesthesiology, \\ Ruijin Hospital, Shanghai Jiaotong University School of Medicine, Shanghai, China. ${ }^{3}$ Department of Environmental \\ and Occupational Health, School of Public Health, and ${ }^{4}$ Aging Institute, Department of Medicine, School of Medicine, \\ University of Pittsburgh, Pittsburgh, Pennsylvania, USA. ${ }^{5}$ Department of Gastroenterology, Ruijin Hospital, Shanghai \\ Jiaotong University School of Medicine, Shanghai, China. ${ }^{6}$ Ohio State University College of Medicine, Columbus, Ohio, \\ USA. ${ }^{7}$ Division of Endocrinology and Metabolism, Department of Medicine, School of Medicine, ${ }^{8}$ McCowan Institute for \\ Regenerative Medicine, and ${ }^{9}$ Vascular Medicine Institute, University of Pittsburgh, Pittsburgh, Pennsylvania, USA.
}

Interleukin-3 (IL-3) receptor $\alpha$ (IL-3R $\alpha$ ) is the $\alpha$ subunit of the ligand-specific IL-3R and initiates intracellular signaling in response to IL-3. IL-3 amplifies proinflammatory signaling and cytokine storm in murine sepsis models. Here we found that RNFT2 (RING finger transmembrane-domain containing protein 2, also TMEM118), a previously uncharacterized RING finger ubiquitin E3 ligase, negatively regulated IL-3-dependent cellular responses through IL-3R $\alpha$ ubiquitination and degradation in the proteasome. In vitro, IL-3 stimulation promoted IL-3R $\alpha$ proteasomal degradation dependent on RNFT2, and we identified IL-3R $\alpha$ lysine 357 as a ubiquitin acceptor site. We determined that LPS priming reduces RNFT2 abundance, extends IL-3R $\alpha$ half-life, and sensitizes cells to the effects of IL-3, acting synergistically to increase proinflammatory signaling. In vivo, IL-3 synergized with LPS to exacerbate lung inflammation in LPS and Pseudomonas aeruginosa-challenged mice; conversely, IL-3 neutralization reduced LPS-induced lung injury. Further, RNFT2 overexpression reduced lung inflammation and injury, whereas Rnft2 knockdown exacerbated inflammatory responses in LPS-induced murine lung injury. Last, we examined RNFT2 and IL-3R $\alpha$ in human lung explants from patients with cystic fibrosis and also showed that IL-3 is elevated in mechanically ventilated critically ill humans at risk for acute respiratory distress syndrome. These results identify RNFT2 as a negative regulator of IL-3R $\alpha$ and show a potential role for the RNFT2/IL-3R $\alpha / I L-3$ axis in regulating innate immune responses in the lung.

Authorship note: YT, TBL, and JE contributed equally to this work.

Conflict of interest: The authors have declared that no conflict of interest exists.

Copyright: ( 2020 , American Society for Clinical Investigation.

Submitted: September 19, 2019 Accepted: January 9, 2020 Published: January 28, 2020.

Reference information: /CI Insight. 2020;5(3):e133652.

https://doi.org/10.1172/jci. insight.133652.

\section{Introduction}

Interleukin-3 (IL-3), a cytokine that regulates hematopoiesis (1), also amplifies cytokine storm in murine sepsis (2), and in septic humans elevated circulating IL-3 is associated with mortality (2). The downstream effects of IL-3 are mediated by binding to its receptor, a complex that contains an $\alpha$ subunit (IL-3 receptor $\alpha$, IL-3R $\alpha$; or CD123) and a $\beta$ subunit (IL-3 receptor $\beta$, IL-3R $\beta$; or CSF2RB) (3). IL-3R $\alpha$-neutralizing antibodies are protective in murine sepsis $(2,4)$, and IL-3 predicts responsiveness to corticosteroid therapy in humans with sepsis (5). Thus, the IL-3/ IL-3R $\alpha$ axis may be a therapeutic target in sepsis and other acute inflammatory disorders. Here we show a previously uncharacterized mechanism regulating IL-3R $\alpha$ abundance through the ubiquitin/proteasome system (UPS), directed by the E3 ligase RING finger transmembrane-domain containing protein 2 (RNFT2).

IL-3-initiated intracellular signaling is dependent on receptor subunits IL-3R $\alpha$ and IL-3R $\beta(1,6)$. The $\beta$ subunit IL-3R $\beta$, also known as the $\beta$-common receptor $(\beta c)$, is a shared receptor subunit that detects several colony-stimulating factor cytokines, including IL-3, GM-CSF, and IL-5 (1, 3, 6). Depending on the stimulus (IL-3, GM-CSF, or IL-5) and the cell type, several intracellular signaling pathways can be activated. The most well-characterized signaling mechanisms include activation of the Janus kinase 2 (JAK2)/signal transducer and activator of transcription 5 (STAT5) pathway and the PI3K pathway (7). Further, activation 
of the NF- $\mathrm{BB}$ pathway through tumor necrosis factor receptor-associated factor 6 (TRAF6) recruitment to the $\beta$ subunit has been described (8). The consequences of these signaling pathways are diverse and context specific, and $\beta c$ cytokines have been implicated in several inflammatory diseases, including allergic asthma, inflammatory bowel disease, multiple sclerosis, and sepsis, among others (9). In murine sepsis, IL-3 potentiates cytokine storm, a phenomenon associated with end organ damage and mortality, and blockade of the IL-3 receptor subunit IL-3R $\alpha$ protects against the deleterious effects of IL-3 (2).

Ubiquitin conjugation is a posttranslational modification of substrate proteins that involves at least 3 enzymes, including a ubiquitin-activating enzyme (E1), a ubiquitin-conjugating enzyme (E2), and a ubiquitin ligase (E3). The E1 activates the C-terminus of ubiquitin by using ATP, which directs ubiquitin to E2. The E3 ligases transfer ubiquitin to substrate proteins, either from a thioester linkage on the E3 received from the E2 or by serving as adapters that bring the substrate in proximity to the E2 for ubiquitin transfer $(10,11)$. Three classes of ubiquitin E3 ligases contribute to protein ubiquitination: RING E3 ligase, HECT E3 ligase, and U-BOX E3 ligase $(11,12)$. The RING E3 ligases contain the most members, and these ligases harbor a unique RING finger domain that consists of 2 zinc finger-type domains (13-15). Several E3 ligases have been shown to regulate innate immunity and inflammation in lung injury (16-21), and here we characterize the E3 ligase RNFT2 as a negative regulator of IL-3R $\alpha$.

In this study, we show the IL-3 receptor subunit IL-3R $\alpha$ is targeted for ubiquitination and degradation by the E3 ligase RNFT2 (also TMEM118). We identify IL-3R $\alpha$ Lys357 as a critical residue regulating IL-3R $\alpha$ stability. In vitro, IL-3 promoted RNFT2-dependent IL-3R $\alpha$ proteasomal degradation, an effect mitigated by LPS priming. Using models of LPS and Pseudomonas aeruginosa (PA) murine lung injury in vivo, we show that exogenous IL-3 administration amplifies inflammation and organ damage, while IL-3 neutralization is protective. Further, in LPS murine lung injury models, RNFT2 overexpression conferred protection from the deleterious effects of IL-3, while Rnft2 knockdown augmented IL-3-dependent effects and lung injury severity. Last, in humans we show that IL-3 was elevated in subjects at risk for acute respiratory distress syndrome (ARDS) and that IL-3R $\alpha$ and RNFT2 abundance are inversely correlated in lung homogenates from lung explants in patients with cystic fibrosis. These data show RNFT2 is a previously unrecognized regulator of IL-3-dependent cellular effects by controlling IL-3R $\alpha$ stability.

\section{Results}

$I L-3 R \alpha$ is degraded in the proteasome in response to $I L-3$. We sought to determine IL-3R $\alpha$ protein stability. We showed IL-3R $\alpha$ expression in murine lung epithelial-12 (MLE-12, hereafter "MLE") cells and RAW264.7 cells (Figure 1A and Supplemental Figure 1A; supplemental material available online with this article; https://doi.org/10.1172/jci.insight.133652DS1). IL-3R $\alpha$ transcript was also present in RNA-Seq experiments from MLE cells (Gene Expression Omnibus accession GSE94680) (22). MLE cells were treated with $\mathrm{CHX}$, and IL-3R $\alpha$ half-life was observed to be approximately 6 hours; additionally, we found CHX-induced IL-3R $\alpha$ degradation was prevented by the proteasome inhibitor MG132 but not by the lysosomal inhibitor leupeptin (Figure 1A). We observed a similar half-life and preservation with MG132 treatment in RAW cells (Supplemental Figure 1A). Because IL-3R $\alpha$ protein is protected by proteasomal blockage, we hypothesized that IL-3R $\alpha$ degradation is regulated by the UPS. Indeed, expression of ubiquitin in MLE cells led to a dose-dependent decrease in IL-3R $\alpha$ protein (Supplemental Figure 1B). Further, IL-3R $\alpha$ protein was retained following coexpression of ubiquitin and MG132 treatment (Figure 1B), an effect not seen with leupeptin treatment.

We next characterized the ubiquitination linkage type because substrate ubiquitination controls several diverse cellular functions (23). We performed Ubiquitin Chain Restriction analysis, in which HIS-tagged IL-3R $\alpha$ was expressed in MLE cells before MG132 treatment, HIS pull-down, and in vitro exposure to deubiquitinases (DuBs) with differential activity to specific ubiquitin chain linkages (24). We observed digestion of IL-3R $\alpha$ polyubiquitination by DuBs with affinity for K48-, K27-, K29-, K33-, K11-, and K63-type linkages (Supplemental Figure 1C). K48-linked polyubiquitination is a common linkage associated with proteasomal degradation $(23,25)$. As an orthogonal approach, we coexpressed IL-3R $\alpha$ and ubiquitin constructs with specific $\mathrm{K} \rightarrow \mathrm{R}$ mutations, before MG132 treatment and IL-3R $\alpha$ pull-down, followed by immunoblotting. We observed that the expression of several mutants, especially K48R and K63R mutants, resulted in reduced polyubiquitinated IL-3R $\alpha$ compared with WT ubiquitin (Supplemental Figure 1D). Taken together, these results suggest IL-3R $\alpha$ ubiquitination is a signal for degradation. Ubiquitin is conjugated to substrate proteins at target lysine $(\mathrm{K})$ residues, and 


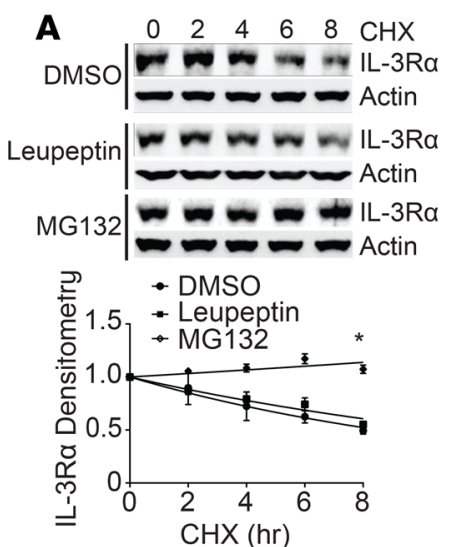

$\mathbf{E}$

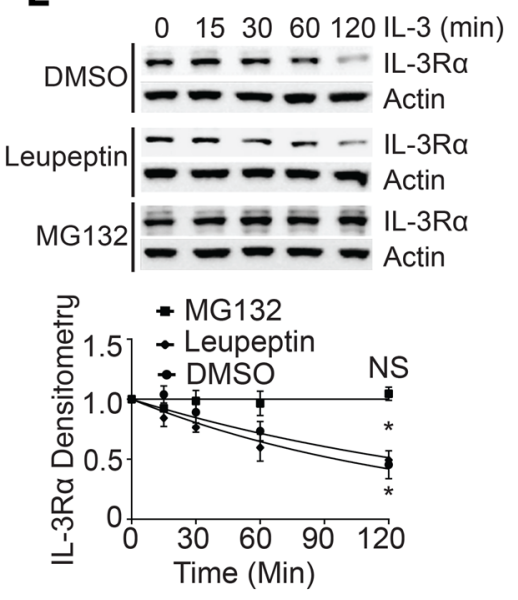

B

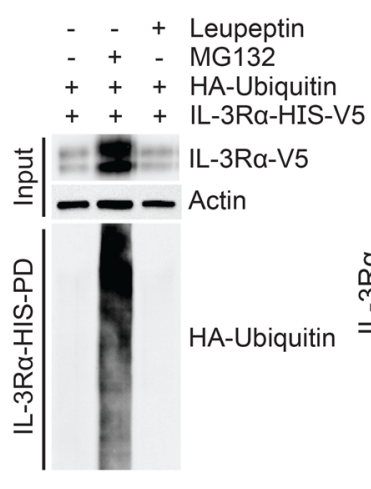

C

D

$\mathbf{F}$
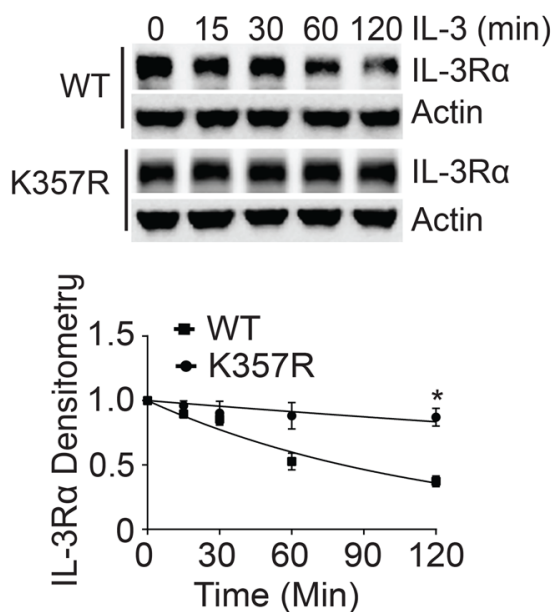

$02550100200 \mathrm{IL}-3$ (ng/mL)

$-\approx-\approx-1 \mathrm{IL}-3 \mathrm{Ra}$

$\sim \sim$ Actin

0153060 120IL-3 (min)

- $2-2 \sim$ IL-3Ra

- $-\infty$ Actin

Figure 1. IL-3R $\alpha$ is degraded in the proteasome in response to IL-3. (A) Immunoblot analysis of IL-3R $\alpha$ protein abundance in MLE cells treated with cycloheximide (CHX) with or without MG-132 or leupeptin in a time-dependent manner. Data and mean \pm SEM of 3 independent experiments. (B) Immunoblotting of IL-3R $\alpha$-V5 HIS pull-down (HIS PD) from MLE cells after coexpression of IL-3R $\alpha$-V5 and HA-ubiquitin and treatment with MG132 or leupeptin. (C) Immunoblot analysis of MLE cells transfected with WT or lysine mutant (K357R) IL-3R $\alpha$ and treated with CHX. Data and mean \pm SEM of 3 independent experiments. (D) Immunoblotting of IL-3R $\alpha$ protein abundance from MLE cells treated with recombinant IL-3 protein (rIL-3) in a dose- or time-dependent manner. (E) Immunoblot analysis of IL-3R $\alpha$ protein amount from MLE cells treated with rIL-3 with or without MG-132 or leupeptin in a time-dependent manner. Data and mean \pm SEM of 3 independent experiments. (F) Immunoblot analysis of IL-3R $\alpha$-V5 protein amount from MLE transfected with WT or K357R mutant IL-3R $\alpha$ and treated with rIL-3 treatment. Data and mean \pm SEM of 3 independent experiments. (C) Immunoblot analysis of MLE cells transfected with IL-3R $\alpha$ or empty plasmid and challenged with or without rIL-3. Data and mean \pm SEM of 3 independent experiments. ${ }^{*} P<0.05,{ }^{* *} P<0.01$, and ${ }^{* * *} P<0.001$ by $F$ test $(\mathbf{A}, \mathbf{C}, \mathbf{E}, \mathbf{F})$ or by 1-way ANOVA with Tukey's post hoc test (C).

we generated IL-3R $\alpha$ lysine-to-arginine $(\mathrm{K} \rightarrow \mathrm{R})$ mutants to identify putative ubiquitin acceptor sites because arginine (R) is unable to accept ubiquitin. We found the IL-3R $\alpha$ K357R mutant protein was resistant to degradation in CHX chase experiments (Figure 1C). Thus, K357 is a candidate ubiquitin acceptor site for IL-3R $\alpha$. These experiments suggest that IL-3R $\alpha$ protein is regulated through ubiquitination leading to proteasomal degradation.

We examined whether IL-3 stimulation would promote proteasomal degradation of IL-3R $\alpha$ and affect its downstream signaling. IL-3R $\alpha$ abundance decreased in a time- and dose-dependent manner following IL-3 stimulation (Figure 1D), an effect that was mitigated by pretreatment with MG132 but not leupeptin (Figure 1E). Additionally, IL-3 stimulation reduced amounts of ectopic IL-3R $\alpha$ WT, but not IL-3R $\alpha$ K357R, in MLE cells (Figure 1F). These results suggest that IL-3R $\alpha$ undergoes ligand-induced degradation in the proteasome. Last, we examined whether IL-3R $\alpha$ affected IL-3-dependent downstream effects. TRAF6-dependent NF-kB activation has been shown as a cellular response to IL-3 (8), and we observed that IL-3 stimulation of MLE cells increased TRAF6 abundance as well as RelB and phospho-P100, proteins involved in the NF- $\mathrm{KB}$ signaling cascade (Figure 1G). Further, overexpression of IL-3R $\alpha$ augmented these effects (Figure 1G).

RING finger E3 ligase RNFT2 regulates IL-3Ra stability and signaling. We next investigated the mechanism involved in IL-3R $\alpha$ protein ubiquitination and degradation. We overexpressed E3 ligases in MLE cells 
A

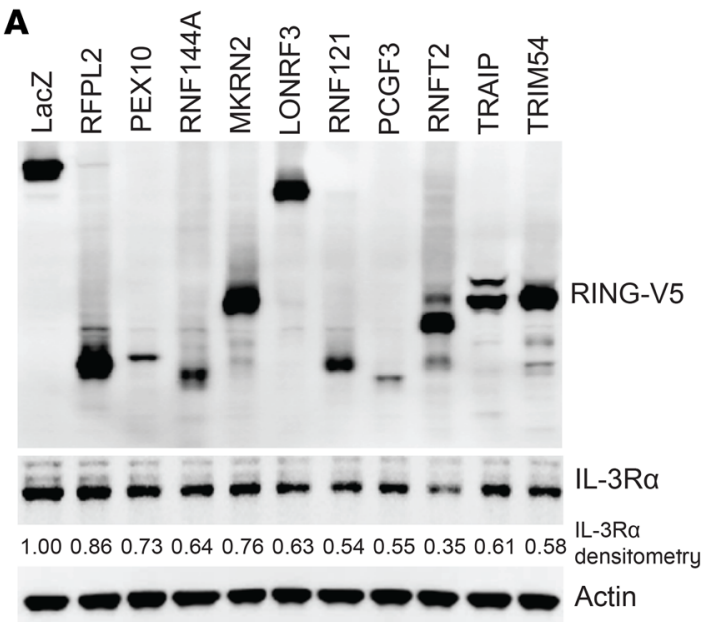

E

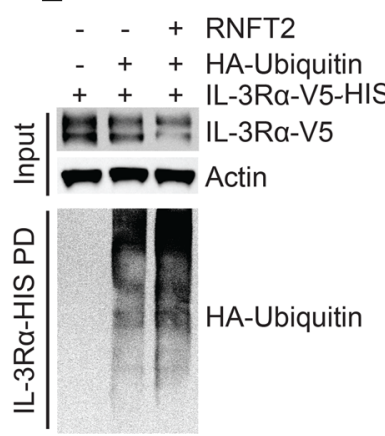

$\mathbf{F}$

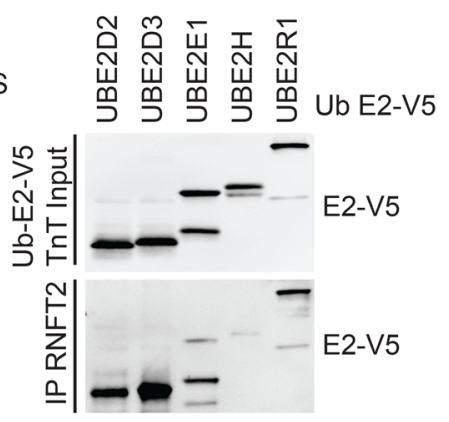

B

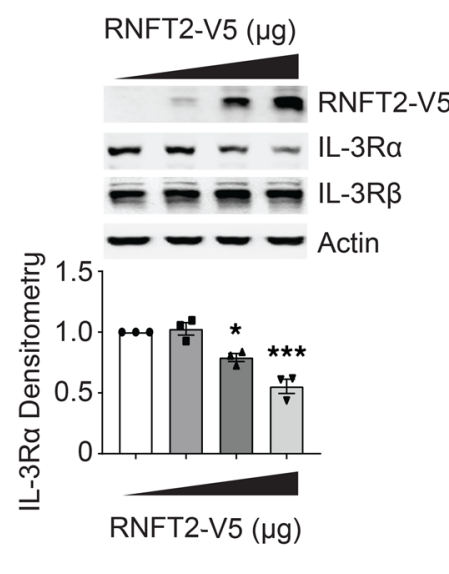

G

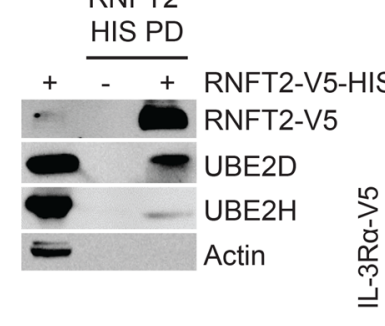

C RNFT2-HA - +

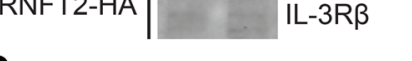

D

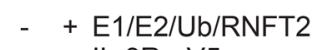

$+\quad+$ IL-3R $\alpha-\mathrm{V} 5$

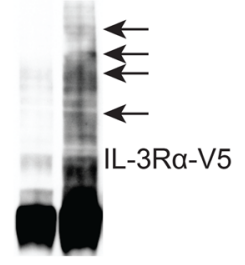

H
$\frac{\mathrm{IL}-3 \mathrm{R} \alpha-\mathrm{V} 5}{\frac{\mathrm{WT}}{-+} \frac{\mathrm{K} 357 \mathrm{R}}{-+ \text { RNFT2-V5 }}}$

RNFT2-V5

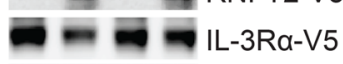

Actin

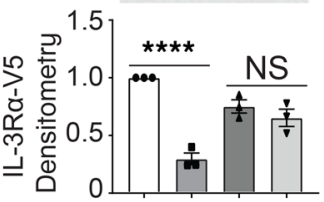

Figure 2. RING finger E3 ligase RNFT2 regulates IL-3R $\alpha$ stability and IL-3 signaling. (A) Immunoblot analysis of IL-3R $\alpha$ from MLE cells transfected with LacZ or 1 of the other 10 randomly selected RING finger E3 ligases. IL-3R $\alpha$ /actin densitometry normalized to LacZ treatment is shown below IL-3R $\alpha$ bands. (B) Immunoblotting of IL-3R $\alpha$ from MLE cells transfected with a dose course of RNFT2. Data and mean \pm SEM of 3 independent experiments. (C) Immunoblot analysis of MLE cells following expression of RNFT2-HA and immunoprecipitation (IP) of RNFT2-HA. (D) Immunoblotting of in vitro ubiquitination assay involving the full complement of ubiquitination machinery (E1, E2, ubiquitin, ATP, $\mathrm{Mg}^{2+}$ ) and RNFT2 protein. (E) Immunoblot analysis of in vivo ubiquitination assay in MLE cells expressing IL-3R $\alpha$-V5-HIS with HA-ubiquitin and RNFT2 before MC132 treatment and HIS tag pull-down of IL-3R $\alpha$-V5-HIS. (F) Immunoblotting of in vitro protein binding assay between V5-tagged ubiquitin E2 enzymes (Ub E2) and immunoprecipitated RNFT2 protein (IP RNFT2). $\mathrm{TnT}$, in vitro transcription and translation. (C) Immunoblot analysis of MLE cells expressing RNFT2-HIS-V5 before HIS pull-down of RNFT2. (H) Immunoblot analysis of MLE cells cotransfected with WT or K357R IL-3R $\alpha$ lysine mutants with or without RNFT2. Data and mean \pm SEM of 3 independent experiments. ${ }^{*} P<0.05,{ }^{* *} P<0.001$, and ${ }^{* * *} P<0.0001$ by 1 -way ANOVA with Dunnett's post hoc test (B) or by 1-way ANOVA with Tukey's post hoc test (H).

$(18,26-28)$ and observed that the uncharacterized E3 ligase RNFT2 decreased IL-3R $\alpha$ protein amounts (Figure 2A), without affecting its mRNA expression (Supplemental Figure 2A). Expression of RNFT2 led to a dose-dependent decrease of IL-3R $\alpha$ protein but not of IL-3R $\beta$ protein (Figure $2 B$ ). We observed that RNFT2 protein associated with IL-3R $\alpha$ in MLE cells by RNFT2 pull-down (Figure 2C). Thus, RNFT2 is a candidate $\mathrm{E} 3$ ligase targeting IL-3R $\alpha$ as a substrate for ubiquitination and degradation.

To confirm that RNFT2 functioned as an E3 ligase to ubiquitinate IL-3R $\alpha$, we performed an in vitro ubiquitination assay. RNFT2 protein in addition to the full complement of ubiquitination machinery was sufficient to ubiquitinate IL-3R $\alpha$ in vitro (Figure 2D). As a complementary approach, we showed that coexpression of RNFT2 with HA-ubiquitin and IL-3R $\alpha$-HIS-V5 led to degradation of IL-3R $\alpha$ protein and increased the HA-ubiquitin signal upon IL-3R $\alpha$-HIS pull-down as compared with control (Figure 2E). As a putative RING finger E3 ligase, RNFT2 functions through its RING domain (Supplemental Figure 2B). Mutation of critical residues within this region led to preservation of IL-3R $\alpha$ protein relative to WT (Supplemental Figure 2C). To further confirm RNFT2 activity as an authentic ubiquitin E3 ligase, we performed in vitro binding assays and observed that RNFT2 indeed bound to several ubiquitin E2 enzymes (Figure $2 \mathrm{~F}$ ). We also confirmed the association of RNFT2 with endogenous ubiquitin-conjugating enzyme E2 D1-3 protein through cell-based pull-down assays (Figure 2G). Last, to demonstrate the specificity of 
A

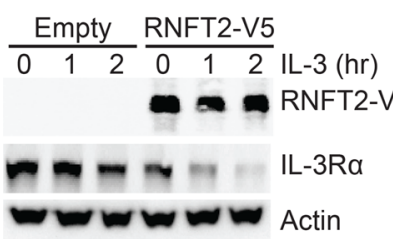

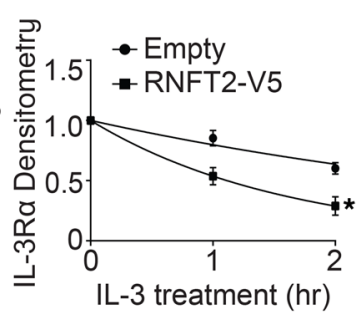

IL-3 treatment (hr)
B

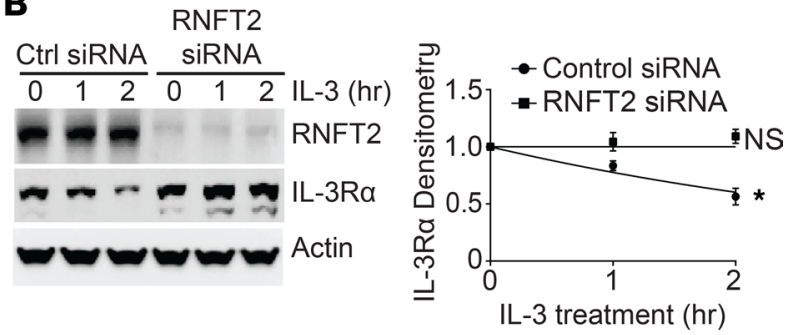

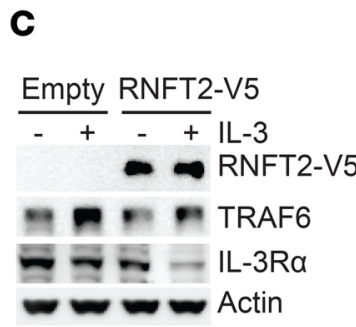
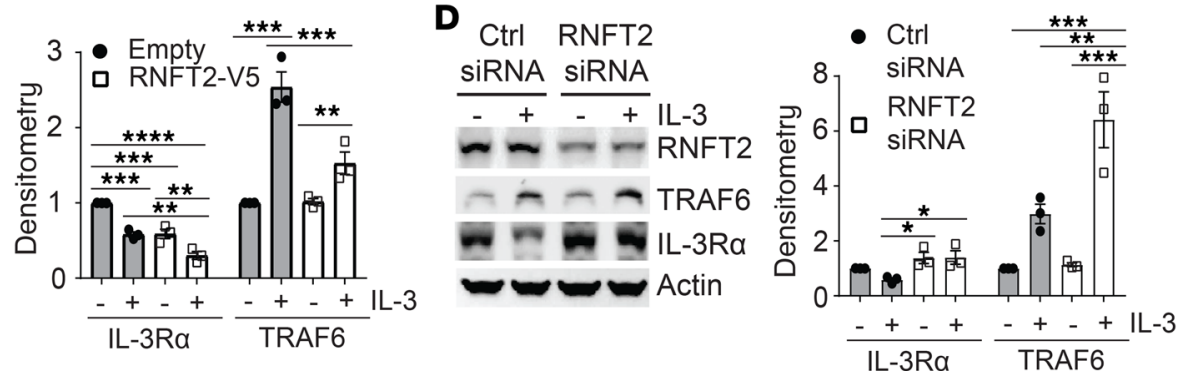

Figure 3. RNFT2 regulates IL-3R $\alpha$ protein levels and IL-3R $\alpha$-dependent inflammatory signaling. (A) Immunoblotting of MLE cells transfected with empty or RNFT2 plasmid before IL-3 treatment. Data and mean \pm SEM of 3 independent experiments. (B) Immunoblot analysis of MLE cells transfected with control siRNA or Rnft2 siRNA before rlL-3 treatment. Data and mean \pm SEM of 3 independent experiments. (C) Immunoblotting of MLE cells transfected with empty or RNFT2 plasmid and challenged with or without rlL-3. Data and mean \pm SEM of 3 independent experiments. (D) Immunoblot analysis of MLE cells transfected with Rnft2 siRNA or control siRNA and then challenged with or without rIL-3. Data and mean \pm SEM of 3 independent experiments. ${ }^{*} P<$ $0.05,{ }^{* *} P<0.01,{ }^{* *} P<0.001$, and ${ }^{* * *} P<0.0001$ by $F$ test $(\mathbf{A}$ and $\mathbf{B})$ or by 1 -way ANOVA with Tukey's post hoc test (C and $\left.\mathbf{D}\right)$.

RNFT2 to target IL-3R $\alpha$, we co-overexpressed RNFT2 with WT or K357R IL-3R $\alpha$. RNFT2 overexpression reduced amounts of IL-3R $\alpha$ WT but not IL-3R $\alpha$ K357R (Figure 2H). Hence, we confirmed that RNFT2 is an authentic E3 ligase that targets IL-3R $\alpha$ as a substrate.

Next, we investigated whether RNFT2 affected IL-3R $\alpha$ abundance and signaling in response to IL-3. RNFT2 overexpression significantly enhanced rIL-3-induced IL-3R $\alpha$ degradation (Figure 3A), whereas Rnft2 knockdown markedly preserved IL-3R $\alpha$ protein with IL-3 stimulation (Figure 3B). We found that ectopic expression of RNFT2 decreased TRAF6 protein amount in rIL-3-stimulated MLE cells (Figure 3C), whereas Rnft2 knockdown significantly increased TRAF6 protein abundance (Figure 3D).

$I L-3$ augments proinflammatory cellular responses to LPS through IL-3R and RNFT2. IL-3 exacerbates the proinflammatory effects of LPS (2). We hypothesized that this effect could be partially mediated through LPS-dependent modulation of IL-3R $\alpha$ stability. LPS treatment in MLE cells led to an increase in IL-3R $\alpha$ protein abundance and a concomitant decrease in RNFT2 protein levels (Figure 4, A and B). Although RNFT2 protein levels did not differ over an 8-hour CHX chase with or without LPS cotreatment, we observed that LPS treatment decreased Rnft2 gene transcription (Supplemental Figure 2, D and E). The LPS-mediated effect on IL-3R $\alpha$ protein levels was specific, as IL-3R $\beta$ protein levels were not affected by LPS (Figure 4C). Further, the increase in IL-3R $\alpha$ protein abundance was independent of changes in Il3ra gene transcription (Figure 4D). We also found that in CHX chase experiments, LPS pretreatment extended IL-3R $\alpha$ half-life (Figure 4E). Cotreatment of LPS with IL-3 increased supernatant cytokine levels of IL-6 and CXCL1 compared with LPS treatment alone, an effect further exacerbated with IL-3R $\alpha$ overexpression (Figure 4, F and G).

We then examined the effect of RNFT2 overexpression or knockdown on cytokine secretion in MLE cells in response to LPS/IL-3. RNFT2 overexpression mitigated the effect of LPS/IL-3 cotreatment on CXCL1 secretion (Figure 4H), while Rnft2 knockdown exacerbated this effect (Figure 4I). Last, we co-overexpressed WT or K357R IL-3R $\alpha$ with an empty vector or RNFT2 and stimulated MLE cells with LPS/IL-3. RNFT2 overexpression mitigated TRAF6 abundance as well as IL-6 and CXCL1 cytokine secretion in cells co-overexpressed with WT IL-3R $\alpha$; however, RNFT2 overexpression had no effect on TRAF6 abundance or IL-6 and CXCL1 cytokine secretion in cells co-overexpressed with K357R IL-3R $\alpha$ (Supplemental Figure 3, A-C). Thus, the degradation-resistant K357R IL-3R $\alpha$ mutant was not affected by RNFT2 overexpression. Taken together, these data suggest that LPS sensitizes cells to IL-3 through stabilization of IL-3R $\alpha$. 
A

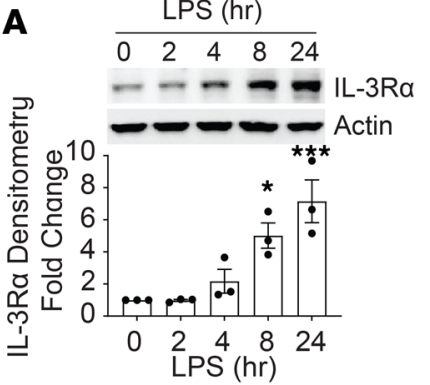

D

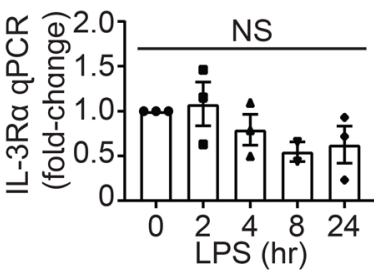

G

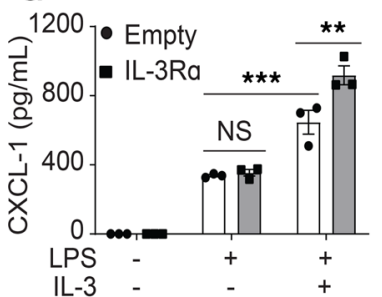

B

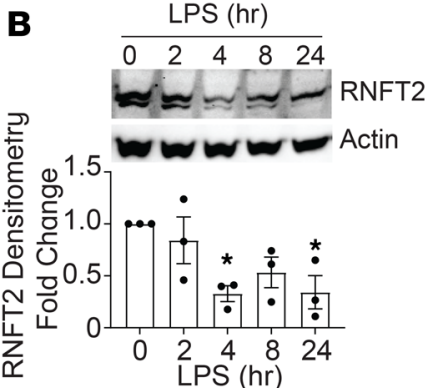

E
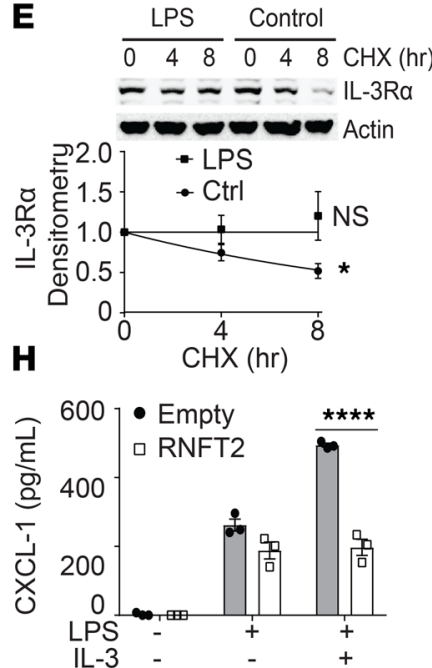

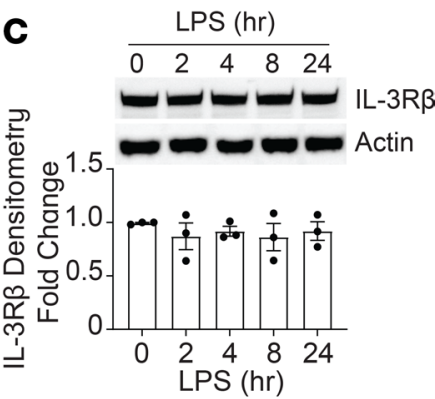

$\mathbf{F}$

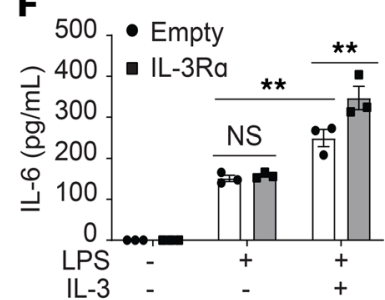

I

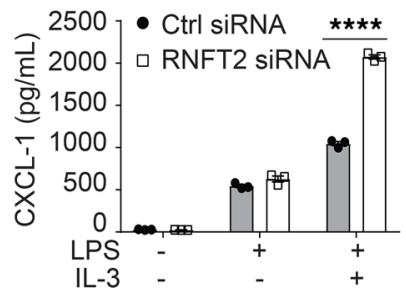

Figure 4. IL-3 augments proinflammatory cellular responses to LPS through IL-3R $\alpha$ and RNFT2. (A-C) Immunoblot analysis of IL-3R $\alpha(\mathbf{A})$, RNFT2 (B), and IL-3R $\beta$ (C) protein abundances in MLE cells treated with LPS at the indicated times. Blots are representative of 3 independent experiments. Quantified data are mean \pm SEM from all experiments $(n=3)$. (D) Reverse transcription-quantitative PCR (RT-qPCR) analysis of IL-3R $\alpha$ mRNA expression in MLE cells treated with LPS at the indicated times. Quantified data are mean \pm SEM from all experiments $(n=3)$. (E) Immunoblot of IL-3R $\alpha$ in MLE cells treated with CHX and control or LPS, as indicated. Blots are representative of 3 independent experiments. Quantified data are mean \pm SEM from all experiments $(n=3)$. ( $F$ and $\mathbf{G})$ ELISA analysis of IL- 6 and CXCL1 in supernatants from MLE cells transfected with IL-3R $\alpha$ or empty plasmid and challenged with LPS and rIL-3, as indicated. Data are mean \pm SEM from all experiments. (H) ELISA analysis of CXCL1 in MLE cells transfected with RNFT2 or empty plasmid before treatment with LPS with or without rlL-3. Data and mean \pm SEM of 3 independent experiments. (I) ELISA analysis of CXCL1 in MLE cells transfected with control siRNA or Rnft2 siRNA before treatment with LPS with or without rlL-3. Data and mean \pm SEM of 3 independent experiments. ${ }^{*} P<0.05$, ${ }^{* *} P<0.01$, ${ }^{* * *} P<0.001$, and ${ }^{* * *} P<0.0001$ by $F$ test (E), by 1-way ANOVA with Dunnett's post hoc test (A-D), or by 1-way ANOVA with Tukey's post hoc test (F-I).

IL-3 augments LPS-induced murine lung injury. To determine whether IL-3 contributed to the pathology of lung injury, we used a clinically relevant model for pneumonia and severe lung injury initiated by intratracheal administration of LPS. After we treated mice with vehicle (PBS), LPS, rIL-3, or their combination intratracheally, we determined lung histology and protein concentration in bronchoalveolar lavage fluid (BALF) 18 hours later. LPS increased BALF protein amounts and the abundance of the inflammatory cytokines IL-6, TNF- $\alpha$, and IL-1 $\beta$, and the damage-associated molecular pattern high mobility group box 1 (HMGB1) in BALF. In contrast, IL-3 treatment alone had only a modest effect on the same parameters. However, cotreatment of IL-3 with LPS dramatically increased the amount of BALF protein and abundance of the inflammatory cytokines IL-6, TNF- $\alpha$, and IL-1 $\beta$, and HMGB1 (Supplemental Figure 4, A-E) compared with LPS alone. BALF cell counts also displayed a similar pattern, with IL-3/LPS cotreatment increasing BALF cell counts most dramatically among treatment groups (Supplemental Figure 4F). Additionally, we observed increased numbers of BALF neutrophils and lymphocytes, but not macrophages, in mice cotreated with IL-3 and LPS compared with PBS, LPS, or IL-3 alone (Supplemental Figure 4, G-I). Lung histopathology was consistent in that IL-3/LPS cotreatment caused the most severe lung injury compared with PBS, LPS, or IL-3 alone (Supplemental Figure 4J). Taken together, these results suggest that IL-3 amplifies LPS-induced lung injury. 


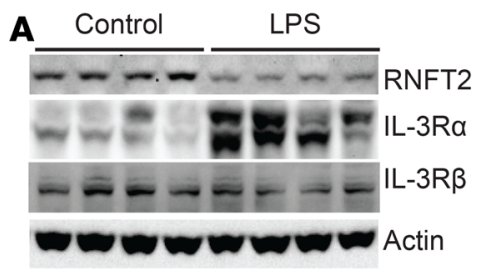

C
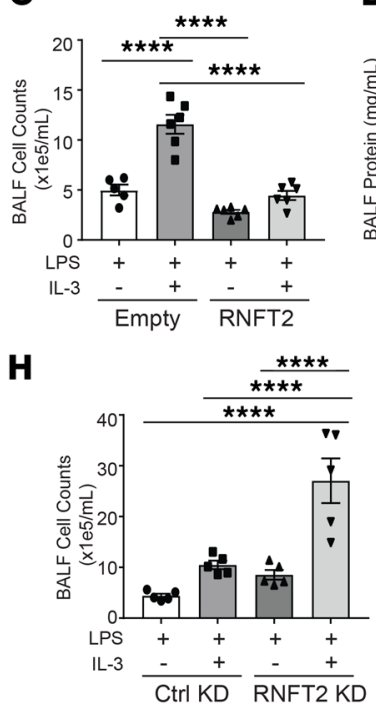
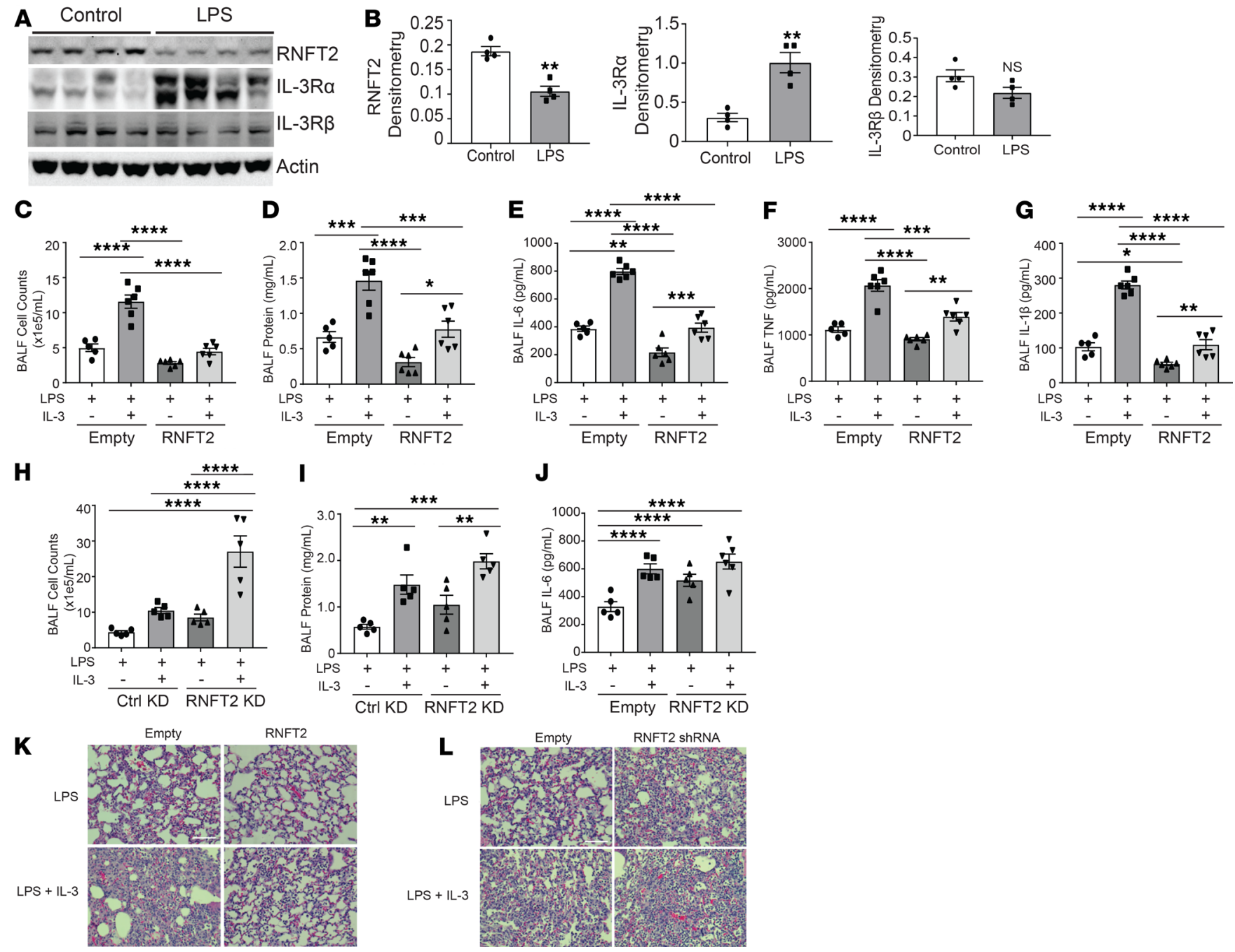

$\mathbf{J}$

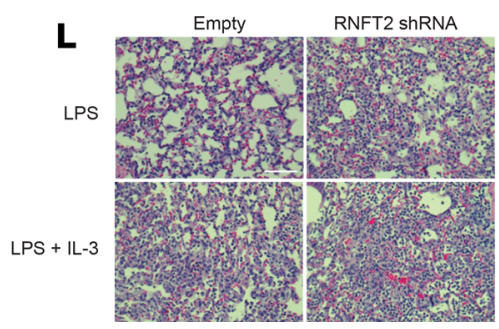

Figure 5. The RNFT2/IL-3R $\alpha$ axis regulates lung innate immunity and inflammation in vivo. (A and B) Immunoblot and densitometry analysis of RNFT2, IL-3R $\alpha$, and IL-3R $\beta$ from mice intratracheally treated with control or LPS, as indicated. Data and mean \pm SEM of 4 mice per group. (C) Cell concentration, (D) total protein analysis, and (E-G) ELISA analysis of (E) IL-6, (F) TNF- $\alpha$, and (C) IL-1 $\beta$ from BALF of mice intratracheally treated with Lenti-empty or Lenti-RNFT2 and then treated with LPS and PBS or rlL-3, as indicated. Data and mean \pm SEM pooled of 3 mice per group are from 2 independent experiments. (H) Cell concentration, (I) total protein analysis, and (J) ELISA analysis of IL-6 from BALF of mice intratracheally treated with Lenti-control shRNA or Lenti-RNFT2 shRNA and then treated with LPS and PBS or rIL-3, as indicated. Data and mean \pm SEM pooled of 3 mice per group are from 2 independent experiments. ( $\mathbf{K}$ and $\mathbf{L})$ Histological analysis of lung samples from mice treated as indicated. Images are representative of all independent experiments. Scale bar: $100 \mu \mathrm{m} .{ }^{*} P<0.05,{ }^{* *} P<0.01,{ }^{* *} P<0.001$, and ${ }^{* * *} P<0.0001$ by Student's $t$ test (B) or by 1-way ANOVA with Tukey's post hoc test (C-J).

IL-3 neutralization reduces inflammation in LPS-induced murine lung injury. In separate experiments, we cotreated mice with LPS and either IgG (control) or a neutralizing antibody against IL-3. We observed that BALF protein; inflammatory cytokines IL- 6 , TNF- $\alpha$, and IL-1 $\beta$; and HMGB1 abundance were all significantly reduced in mice treated with anti-IL-3 compared with IgG-treated control mice (Supplemental Figure 5, A-E). Further, blocking IL-3 antibody treatment reduced BALF cell counts and neutrophils but had no effect on macrophage or lymphocyte counts (Supplemental Figure 5, F-I). Blocking IL-3 antibody cotreatment reduced the severity of lung injury in histopathological sections (Supplemental Figure 5J). Thus, IL-3 may drive inflammation in LPS-induced lung injury.

The RNFT2/IL-3Ra/IL-3 axis regulates lung inflammation in vivo and in vitro. We next examined the role of RNFT2 during acute lung injury. RNFT2 is an uncharacterized E3 ligase. We first performed IHC on mouse lung sections and confirmed RNFT2 was expressed in the mouse lung (Supplemental Figure 6A). We then measured RNFT2, IL-3R $\alpha$, and IL-3R $\beta$ protein amounts in lung homogenates after intratracheal LPS treatment and observed that RNFT2 abundance decreased, IL-3R $\alpha$ abundance increased, and IL-3R $\beta$ amounts were unchanged compared with control mice (Figure 5, A and B). We also observed 
A

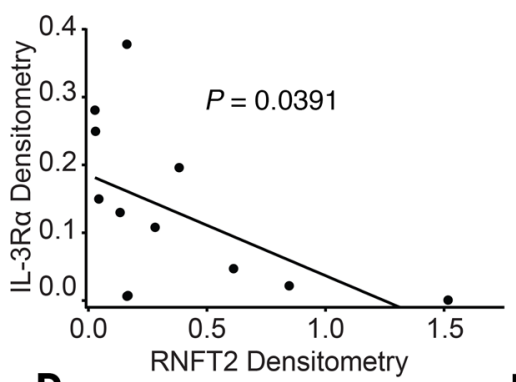

D

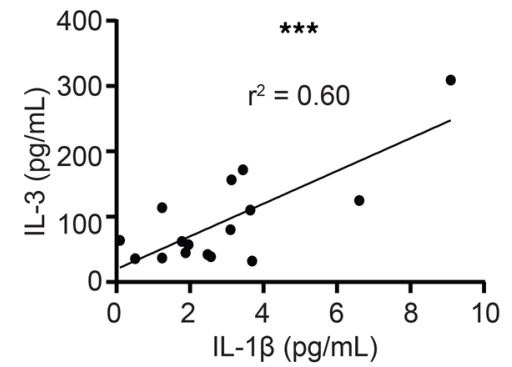

B

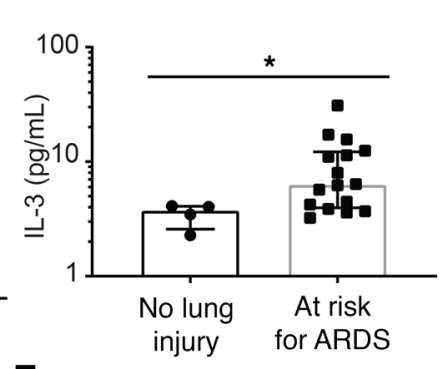

\section{E}

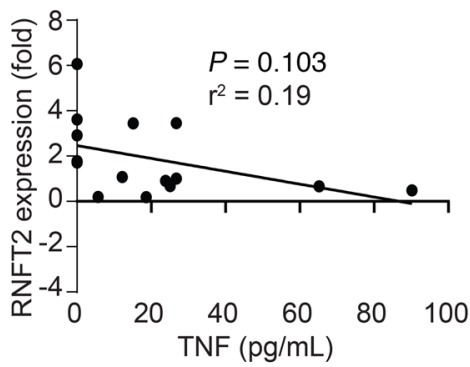

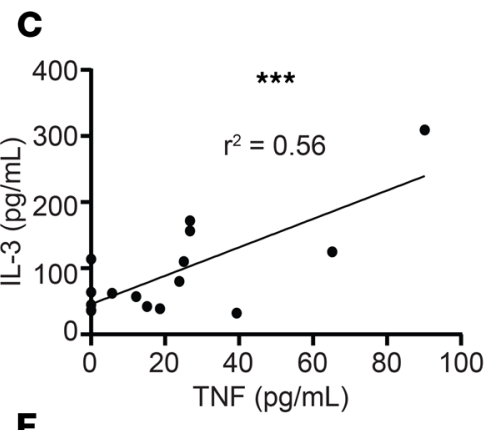

$\mathbf{F}$

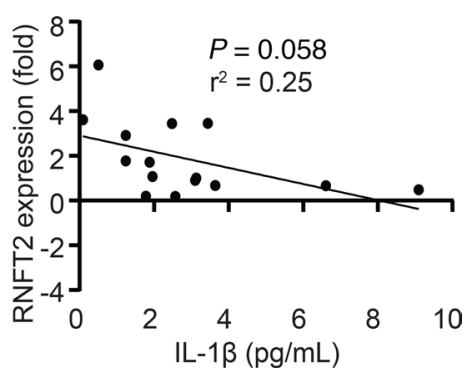

Figure 6. The IL-3R $\alpha /$ RNFT2 signaling axis is relevant in human lung disease. (A) "Densitometric analysis of RNFT2 and IL3R $\alpha$ immunoblotting from Human CF subjects lung explants with Pseudomonas colonization $(n=12)$. (B) ELISA analysis of plasma IL-3 in mechanically ventilated patients without lung injury $(n=4)$ or at risk for ARDS (ARFA; $n=16$ ). Subject classification was blinded to the operator. Bars and error bars represent median and IQR, respectively. Data were analyzed by Mann-Whitney $U$ test. ${ }^{*} P<0.05$. (C and $\left.\mathbf{D}\right)$ Linear regression of plasma IL-3 versus plasma TNF- $\alpha$ (C) and IL-1 $\beta$ (D) in subjects ARFA $(n=16) ;{ }^{* *} P<0.001$; F-test of Linear Regression, Pearson's R ${ }^{2}$ goodness of fit. (E and $\left.\mathbf{F}\right)$ Linear regression of RNFT2 gene expression normalized to control patients versus plasma TNF- $\alpha(\mathbf{E})$ and IL-1 $\beta(\mathbf{F})$ in subjects ARFA $(n=15)$.

increased IL-3 in the BALF of LPS-treated mice compared with controls (Supplemental Figure 6B) To validate these observations in another infectious acute lung injury model, we treated mice with PA; measured abundance of RNFT2, IL-3R $\alpha$, and IL-3R $\beta$; observed a similar pattern of protein signal and of IL-3 signal in murine BALF (Supplemental Figure 6, C-G).

To determine the role of RNFT2 in LPS/IL-3-induced lung injury, we infected mice intratracheally with empty lentivirus or lentivirus encoding $R n f t 2$ and subsequently challenged them with LPS with or without rIL3. Rnft2 gene transfer significantly reduced lung inflammation and injury caused by LPS and rIL-3 cotreatment as shown by decreased BALF cell counts, protein concentrations, cytokine release, and lung infiltrates (Figure $5, \mathrm{C}-\mathrm{G}$, and $\mathrm{K})$. Specifically, the BALF leukocyte differential revealed that the total decrease in inflammatory cells was mostly from neutrophils and lymphocytes but not macrophages (Supplemental Figure 7, A-C).

Next, we administered lentivirus intratracheally encoding control shRNA or Rnft2 shRNA to mice and then exposed them to LPS with or without rIL-3. Rnft2 knockdown significantly enhanced lung inflammation and injury caused by LPS and rIL-3 treatment as shown by increased BALF cell counts, protein concentrations, cytokine release, and lung infiltrates (Figure 5, H-J, and L). Specifically, the differential cell counts of BALF revealed that the total increase in inflammatory cells was mostly from neutrophils and lymphocytes but not macrophages (Supplemental Figure 7, D-F). These results suggest that RNFT2 modulates lung inflammation in vivo.

The IL-3/IL-3R /RNFT2 axis is relevant in human inflammatory lung disease. To determine protein expression patterns of IL-3R $\alpha$ and RNFT2 in clinically relevant human disease, we immunoblotted parenchymal explant lung samples from human cystic fibrosis (CF) patients colonized with PA for RNFT2 and IL-3R $\alpha$ protein amounts, and we quantified their relative expression normalized to tubulin. We observed a significant negative correlation between RNFT2 and IL-3R $\alpha$ protein signal (Figure 6A) (Pearson's $r^{2}=0.278$ ). These studies suggest that the RNFT2/IL-3R $\alpha$ pathway may be relevant in response to PA infection in patients, where decreased amounts of RNFT2 result in reciprocally increased IL-3R $\alpha$ abundance.

Circulating IL-3 may be a potentiator of cytokine storm, and in a cohort of 60 patients with septic shock, high IL-3 (>89.4 pg/mL) amounts were associated with mortality. In this cohort, there was no significant difference in circulating IL-3 protein abundance at day 0 versus day 1 or day 2 following sepsis diagnosis (2). However, in a separate cohort, IL-3 was significantly elevated in patients with sepsis relative to healthy controls (29). We sought to determine whether circulating IL-3 differed in a more homogenous population at risk 
Table 1. Baseline characteristics of patients in the Acute Lung Injury Biospecimen Repository analyzed for plasma IL-3 levels

\begin{tabular}{|c|c|c|}
\hline Variable & No lung injury & At risk for ARDS \\
\hline $\mathbf{N}$ & 4 & 16 \\
\hline Age, mean (SD), yr & $64.9(12.8)$ & $51.8(14.8)$ \\
\hline Males, $N(\%)$ & $4(100)$ & $10(62.5)$ \\
\hline SOFA score, median (IQR) & $5.0(4.5-5.5)$ & $7.0(5.75-9.25)$ \\
\hline $\mathrm{PaO}_{2} / \mathrm{FiO}_{2}$ ratio, mean (SD), mmHg & $281.5(187.7)$ & $217.1(101.5)$ \\
\hline WBCs, mean (SD), × $\mathbf{1 0}^{-9}$ per liter & $14.5(10.8)$ & $13.6(8.7)$ \\
\hline ICU LOS, median (IQR), d & $4.5(3.75-7.25)$ & $6.5(4.75-9.75)$ \\
\hline 30-Day mortality, N (\%) & $1(25)$ & $2(12)$ \\
\hline
\end{tabular}

Data are presented as mean (SD) for normally distributed continuous variables, median (IQR) for non-normally distributed continuous variables, and $N$ (\%) for categorical variables. SOFA, sequential organ failure assessment; $\mathrm{PaO}_{2}$, partial pressure of arterial oxygen; $\mathrm{FiO}_{2}$, fractional inhaled concentration of oxygen; WBCs, white blood cells; ICU LOS, intensive care unit length of stay.

for ARDS because a subset of patients at risk for or with ARDS have excessive inflammation and cytokine storm in the lung $(30,31)$. We assayed IL-3 in plasma from mechanically ventilated subjects at risk for ARDS versus mechanically ventilated subjects without lung injury (Table 1 and Supplemental Table 1). Subjects at risk for ARDS had increased plasma IL-3 compared with subjects without lung injury (Figure 6B). Further, IL-3 levels were associated with other plasma proinflammatory cytokines in this cohort, including TNF- $\alpha$ and IL-1 (Figure 6, C and D). We also measured RNFT2 gene expression through RT-qPCR from white blood cell pellets from the same subjects but found no significant correlations to the same proinflammatory cytokines (Figure 6, E and F). Thus, high circulating IL-3 may be an additional biomarker to identify subjects at risk for ARDS with excessive lung inflammation. Further studies with increased power are warranted to determine whether IL-3 could be a new biomarker in patients with or at risk for ARDS.

In summary, our studies demonstrate IL-3R $\alpha$ stability is regulated through the UPS and is dependent on the previously uncharacterized E3 ligase RNFT2. These findings may have implications for pulmonary inflammatory disorders, including acute lung injury. We characterize RNFT2 as an authentic E3 ligase that directs IL-3R $\alpha$ ubiquitination and degradation and identify Lys357 on IL-3R $\alpha$ as a critical residue regulating its stability because K357R IL-3R $\alpha$ mutants are resistant to RNFT2-directed degradation. In vivo, we show (a) IL-3 exacerbated LPS-induced lung injury, (b) IL-3 neutralization reduced inflammation in LPS-induced lung injury, (c) RNFT2 overexpression was protective in LPS/IL-3-induced lung injury and mitigated the effects of IL-3, and (d) Rnft2 knockdown exacerbated LPS/IL-3-induced lung injury and exacerbated the effects of IL-3.

\section{Discussion}

IL-3 has recently been implicated in the pathogenesis of several inflammatory disorders, including collagen-induced arthritis (32), autoimmune encephalitis (33), and lupus nephritis (34). In some of these models, IL-3 blockade reduced disease severity scores and was associated with decreased systemic inflammatory markers, whereas administration of IL-3 exacerbated these effects $(33,34)$. Our findings are in line with these studies because we show that IL-3 blockade reduced lung injury and inflammatory markers in a murine model of severe lung injury and that addition of IL-3 exacerbated organ damage. Our results are also consistent with those of Weber et al., who demonstrated that IL-3 alone is not sufficient to induce a systemic inflammatory response, but rather serves to "amplify" signaling initiated by an initial insult (cecal ligation and puncture or intratracheal LPS) (2). Here we extensively studied the role of the IL-3/IL-3R $\alpha$ axis in lung innate immunity and inflammation. We found IL-3 amounts were increased in BALF of mice subjected to LPS or PA treatment, and rIL-3 cotreatment with LPS drastically enhanced proinflammatory cytokine release and immune cell activation in murine lungs. We observed IL-3 treatment synergizes with bacterial pathogen-associated molecular pattern (PAMP) exposure to strongly activate downstream signaling through NF- $\mathrm{KB}$ activation, leading to proinflammatory cytokine release. Our model is consistent with the "second-hit" theory or multicausal effect mentioned in previous studies $(35,36)$.

IL-3 classically signals through the JAK/STAT pathway (9) but has also been linked to NF-kB through TRAF6 (8). TRAF6 is 1 of the 6 well-characterized TRAF proteins, which are critical contributors to inflammatory, innate, and adaptive immune responses $(28,37,38)$. Specifically, TRAF6 is a key mediator of TLR 
signaling and downstream NF-кB signaling (39-41). We found TRAF6 increased in MLE cells treated with rIL3 , suggesting TRAF6 is a candidate contributor to the synergy of IL-3 and NF- $\mathrm{kB}$ activation. We also observed that ectopic expression of the IL-3R $\alpha$ significantly increased TRAF6 amount and enhanced cytokine release upon IL-3/LPS cotreatment (Figure $1 G$ and Figure 4, F and G). These studies suggest that IL-3 along with its receptor, IL-3R $\alpha$, synergize with bacterial PAMP exposure through downstream NF- $\mathrm{B}$ signaling.

In this study, we found that IL-3R $\alpha$ protein abundance is regulated posttranslationally through ubiquitination. Protein ubiquitination is a universal cellular mechanism controlling protein amount and innate immunity $(20,42-45)$. Recently, we have shown that several E3 ligases regulate substrate ubiquitination in pulmonary inflammatory conditions $(17,18,21)$. During systemic insults induced by infection, alterations of E3 ligase activity or targeting can affect substrate protein abundance and their downstream effector functions. We showed that intratracheal treatment of LPS or PA in vivo increases substrate IL-3R $\alpha$ protein amount and that LPS treatment of MLE cells increases IL-3R $\alpha$. Lung tissue from PA-treated mice showed decreased abundance of the putative E3 ligase RNFT2, with a correlative increase in IL-3R $\alpha$ protein, suggesting the exogenous insult manipulated RNFT2 protein amount and thus the protein stability of its substrate IL-3R $\alpha$. We characterized RNFT2 as an authentic E3 ligase by showing its interaction with the E2 enzyme UBE2D in vitro. We further found that RNFT2 regulated the rIL-3-induced increase in TRAF6 protein and affected CXCL1 secretion in MLE cells. Specifically, ectopic expression of RNFT2 in MLE cells significantly suppressed cotreatment-induced (LPS with rIL-3) cytokine release, whereas Rnft2 knockdown enhanced CXCL1 secretion (Figure 4, H and I). RNFT2 gene transfer and knockdown in vivo also has a similar phenotype in cytokine release and immune cell activation, such as neutrophils, in lung. Our data imply that RNFT2 functions as an inflammatory suppressor protein that controls IL-3 signaling in innate immunity, and the RNFT2/ IL-3R $\alpha$ axis may be important in the lung innate immune response chain.

This study also provided 2 additional human translational links that suggest the IL-3/IL-3R $\alpha$ signaling axis may play a role in human inflammatory lung diseases. We show that plasma IL-3 protein amounts are increased in humans at risk for ARDS compared with mechanically ventilated subjects without lung injury. These findings warrant examination in larger lung injury cohorts and suggest that IL-3 may be a previously unrecognized cytokine associated with excessive inflammation. We also examined protein abundance of RNFT2 and IL-3R $\alpha$ in explanted lung tissue from patients with chronic pseudomonas infection. We discovered there was an inverse correlation between RNFT2 and IL-3R $\alpha$ protein amount, suggesting that our newly described mechanism of RNFT2-dependent IL-3R $\alpha$ degradation may be operant in humans with chronic infection. Further studies are warranted to delineate the role of RNFT2 in these inflammatory disorders.

There are limitations to these studies. Although our in vivo work clearly identifies a role for IL-3 in LPS and PA murine acute lung injury, we do not identify the cell type responsible for IL-3 secretion. In murine sepsis models, innate response activator (IRA) B cells are thought to be responsible for IL-3 secretion $(2,46)$. Further, T cells and B cells are classically regarded as the main IL-3 producers in other models of disease (7, 9, 32, 33). Whether IRA B cells are also responsible for IL-3 secretion in acute lung injury models is an active area of investigation. Further, whether these cells express RNFT2 is also unknown. Recent single-cell RNA-sequencing studies on murine airway cells showed no detection or extremely low levels of $I l 3$ gene expression, which was not significantly altered among different airway cell types (47-49), suggesting that lung cells are not a primary producer of IL-3. Additionally, although we show in vitro that IL-3R $\alpha$ abundance increases and RNFT2 abundance decreases in LPS-stimulated MLE cells, the changes in RNFT2 and IL-3R $\alpha$ abundance in lung homogenates in vivo during lung injury could also be explained by differences in infiltrating immune cells between control and LPS/PA-treated mice. Thus, although our in vitro work provides mechanistic insight into RNFT2/IL-3R $\alpha$, additional work remains to further characterize this pathway in vivo.

We used the MLE-12 cell line to characterize IL-3R $\alpha$ stability and test the role of RNFT2 in modulating IL-3R $\alpha$-dependent downstream effects. MLE-12 cells are an immortalized cell line that closely resembles, but does not fully mirror, type II alveolar epithelial cells. We have identified several E3 ligase/substrate interactions relevant in pulmonary inflammatory conditions using MLE-12 cells (28, 50-52). Future studies will focus on examining RNFT2/IL-3R $\alpha$ expression in other pulmonary cell types, including primary human cells. We chose to examine NF-אB signaling (TRAF6 abundance, IL-6 secretion, CXLC-1 secretion) because our laboratory has previously shown a role for TRAFs in experimental lung injury (28), and IL-3 signaling has been linked to TRAF6 recruitment (8). However, whether RNFT2 also has similar effects on IL-3R $\alpha$-dependent signaling in immune cells is unknown. 
Last, our in vitro findings provide a framework to interrogate the role of RNFT2 in vivo in pathological disease states where IL-3 is implicated. Related to our studies, it will be critical to determine which cell types are responsible for mediating the effects we observed after intratracheal lentiviral RNFT2 overexpression or knockdown. More broadly, these findings could have implications in other disease states where IL-3R $\alpha$ expression is altered. For example, IL-3R $\alpha$ is overexpressed in several hematologic malignancies and is the basis for novel therapies including chimeric antigen receptor $\mathrm{T}$ cell therapies $(53,54)$. The role of RNFT2 in these conditions is unknown, and one could postulate that reduced RNFT2 expression or function could be partially responsible for aberrant IL-3R $\alpha$ expression. Further, pharmacological targeting of RNFT2 with a small molecule inhibitor could enhance IL-3R $\alpha$ expression.

In conclusion, we showed that IL-3 synergizes with LPS to exacerbate lung inflammatory injury, resulting in increased NF- $\mathrm{kB}$ activation and lung injury. This synergy proceeds through the IL-3R $\alpha$ subunit, whose protein stability is weakened by IL-3 treatment but preserved by LPS exposure. IL-3R $\alpha$ protein is processed by the UPS, and the previously uncharacterized RING E3 ligase, RNFT2, targets IL-3R $\alpha$ for proteasomal degradation by ubiquitinating it at Lys357. The RNFT2/IL-3R $\alpha$ axis controls proinflammatory cytokine and immune cell release in murine lung inflammation models. Taken together, this study characterized a regulatory mechanism involving RNFT2-targeted degradation of the IL-3 cytokine receptor IL-3R $\alpha$.

\section{Methods}

Reagents. Anti-IL-3R $\alpha$ antibodies (MBS2542745; sc-74522) were obtained from MYBioSource and Santa Cruz Biotechnology. Anti-IL-3R $\beta$ (MBS2534790) antibody was from MYBioSource. Monoclonal antiHA-tag (clone 6E2) (2367S RRID AB_10691311), anti-TRAF6 (8028, RRID AB_10858223), anti-RelB (10544, RRID AB_2797727), and anti-phospho-P100 (4810, RRID AB_659925) were from Cell Signaling Technology. Anti-V5 antibody (R960, RRID AB_159298) was from Invitrogen, Thermo Fisher Scientific. Anti-actin antibody (A5441, RRID AB_476744) and leupeptin (L2884) were from MilliporeSigma. Goat anti-rabbit IgG-HRP (170-6515 RRID AB_11125142), goat anti-mouse IgG-HRP (170-6516 RRID AB_11125547), and CFX96 Touch Real-Time qPCR (1855196) were from Bio-Rad. MG-132 (F1100) was from UBPBio. Recombinant murine IL-3 was from PeproTech. Murine CXCL1 (DY453) and murine TNF- $\alpha$ (DY410) ELISA kits were from R\&D Systems. Murine IL-1 $\beta$ (catalog 88-7261) and murine IL-6 (catalog 88-7064, RRID AB_2574986) ELISA kits were from eBioscience. SYBR Select Master Mix (4472918) was from Applied Biosystems, Thermo Fisher Scientific. QuikChange II XL Site-Directed Mutagenesis Kit (200522) was from Agilent Technologies. High-Capacity RNA-to-cDNA Kits (4387406) were from Applied Biosystems, Thermo Fisher Scientific.

Cell culture. MLE (CRL-2110) cells were obtained from ATCC and cultured in Dulbecco's modified Eagle medium-F12 (Gibco, Thermo Fisher Scientific) with 10\% fetal bovine serum (DMEM-F12-10). For RNFT2 overexpression in MLE cells, electroporation was used, and 24 or 48 hours later, cells were treated with stimulators. For RNFT2 knockdown, scramble siRNA and RNFT2 siRNA were used to transfect MLE cells for 48 hours by electroporation. Cell lysates were collected in lysis buffer $(150 \mathrm{mM} \mathrm{NaCl}, 50$ $\mathrm{mM}$ Tris, $1.0 \mathrm{mM}$ EDTA, $2 \mathrm{mM}$ dithiothreitol, $0.025 \%$ sodium azide, and $1 \mathrm{mM}$ phenylmethylsulfonyl fluoride) and prepared by brief sonication at $4^{\circ} \mathrm{C}$. See complete unedited blots in the supplemental material. Additionally, MLE cells were treated with cycloheximide $(100 \mu \mathrm{g} / \mathrm{mL})$ for half-life studies. RAW264.7 cells were from ATCC (TIB-71) and cultured in DMEM with 10\% fetal bovine serum. HEK293T cells were from ATCC and were cultured in DMEM with 10\% fetal bovine serum. THP-1 cells were from ATCC (TIB-202). PBMCs and THP-1 cells were cultured in RPMI medium 1640 (Gibco, Thermo Fisher Scientific) with $10 \%$ fetal bovine serum. Pseudomonas aeruginosa (PA103) was from ATCC (catalog 29260).

$R T-q P C R$. Total RNA was isolated and reverse transcription was performed followed by real-time quantitative PCR with SYBR Green qPCR mixture as described (18).

In vitro ubiquitin conjugation assays. The assay was performed in a volume of $20 \mu \mathrm{L}$ containing $50 \mathrm{mM}$ Tris at $\mathrm{pH}$ 7.6, $5 \mathrm{mM} \mathrm{MgCl}_{2}, 0.6 \mathrm{mM}$ DTT, $2 \mathrm{mM}$ ATP, $10 \mu \mathrm{M} \mathrm{MG132,} 100 \mathrm{nM}$ ubiquitin-activating enzyme, $0.5 \mu \mathrm{M}$ UBE2D, $2 \mu \mathrm{M}$ ubiquitin, and $1 \mu \mathrm{M}$ ubiquitin aldehyde. TnT-coupled reticulocyte in vitro-synthesized proteins (tagless RNFT2 and HIS-purified IL-3R $\alpha$-V5) and reaction products were processed for V5 immunoblotting.

Plasmid transfection. For protein overexpression in MLE-12 cells, cells were nucleofected using Nucleofector 2B (Amaxa), program T-13. For protein overexpression in HEK293T cells (ATCC), XtremeGENE transfection reagents were used following the manufacturer's protocol. Expression was confirmed by Western blotting. 
Recombinant DNA constructs. Total RNA was isolated from untreated human PBMCs using RNeasy Mini Kit (QIAGEN) and reverse-transcribed to cDNA using High-Capacity RNA-to-cDNA Kits. RNFT2 cDNA were PCR amplified, isolated, and sequence confirmed before PCR cloning into pcDNA3.1D-V5HIS vector. Deletion mutants were generated by PCR cloning. Point mutants of RNFT2 and IL-3R $\alpha$ were generated using the QuikChange II XL kit, per the manufacturer's protocol. pRK5-HA-UbiquitinWT was a gift from Ted Dawson (Addgene plasmid 17608, ref. 55) (Johns Hopkins University School of Medicine, Baltimore, Maryland, USA).

In vitro protein binding assays. RNFT2 protein was immunoprecipitated from $1 \mathrm{mg}$ MLE cell lysate using 1:100 dilution of RNFT2 antibody (rabbit; MyBioSource [MBS3209802]) for 4 hours at $25^{\circ} \mathrm{C}$ in IP buffer (50 mM Tris- $\mathrm{HCl}$ at $\mathrm{pH} 7.6,150 \mathrm{mM} \mathrm{NaCl}, 0.25 \% v / v$ Triton X-100) and coupled to protein A/G agarose resin for an additional hour. V5-ubiquitin E2 enzymes $(20 \mu \mathrm{L})$ were synthesized in vitro using TnT translation kits (Promega) for 90 minutes at $30^{\circ} \mathrm{C}$. RNFT2-bound resin was then incubated with the in vitro synthesized proteins for 18 hours at $4^{\circ} \mathrm{C}$. Following binding, resin was washed with IP buffer, and eluted by dilution in denaturing loading buffer, with a final $1 \times$ formulation of $50 \mathrm{mM}$ Tris- $\mathrm{HCl}$ at $\mathrm{pH} 6.8,2 \%$ SDS, $10 \%$ glycerol, and $100 \mathrm{mM} \mathrm{DTT}$ and incubation at $88^{\circ} \mathrm{C}$ for 5 minutes before immunoblotting analysis.

IP assays. MLE cells were cultured and treated as indicated before collection. Cell pellets were lysed with IP buffer ( $0.25 \%$ Triton X-100 in $1 \times$ PBS at $\mathrm{pH} 7.6,0.025 \%$ sodium azide, and $1 \mathrm{mM}$ phenylmethylsulfonyl fluoride) on ice. Lysates were prepared by brief sonication at $4^{\circ} \mathrm{C}$. Insoluble cellular debris was precipitated through centrifugation at 15,000 RCF for 10 minutes at $4^{\circ} \mathrm{C}$. Lysate supernatant was normalized for protein concentration. Supernatants were exposed to a 1:50 dilution of the indicated antibody for 3 hours at $25^{\circ} \mathrm{C}$. Immunoprecipitated protein was captured with $10 \mu \mathrm{L}$ magnetic protein $\mathrm{A} / \mathrm{G}$ resin for 1 hour before 2 rounds of washing with IP buffer $(1 \mathrm{~mL})$. Protein was eluted by dilution in denaturing loading buffer, with a final $1 \times$ formulation of $50 \mathrm{mM}$ Tris- $\mathrm{HCl}$ at $\mathrm{pH} 6.8,2 \%$ SDS, $10 \%$ glycerol, and $100 \mathrm{mM}$ DTT and incubation at $88^{\circ} \mathrm{C}$ for 5 minutes. Eluted samples were resolved by SDS-PAGE and subjected to immunoblotting.

HIS pull-down assays. MLE cells were cultured and treated as indicated before collection. Lysate was recovered in pull-down buffer $(0.25 \%$ Triton $\mathrm{X}-100$ in $1 \times \mathrm{PBS}$ at $\mathrm{pH} 8.0,30 \mathrm{mM}$ imidazole, and $1 \mathrm{mM}$ phenylmethylsulfonyl fluoride) on ice, before brief sonication and sedimentation by centrifugation $(15,000$ RCF, 10 minutes, $4^{\circ} \mathrm{C}$ ). Lysate supernatants were subjected to HIS pull-down by $10 \mu \mathrm{L}$ magnetic HisPur resin for 1 hour before 2 rounds of washing with pull-down buffer $(1 \mathrm{~mL})$. Protein was eluted by dilution in denaturing loading buffer, with a final $1 \times$ formulation of $50 \mathrm{mM}$ Tris- $\mathrm{HCl}$ at $\mathrm{pH} 6.8,2 \% \mathrm{SDS}, 10 \%$ glycerol, and $100 \mathrm{mM}$ DTT and boiling for 5 minutes. Eluted samples were resolved by SDS-PAGE and subjected to immunoblotting.

Animal studies. C57BL/6J male mice were purchased from the Jackson Laboratories. Animals were between 7 and 9 weeks of age and around $25 \mathrm{~g}$ in weight at the time of experiments. All procedures were approved by the University of Pittsburgh Institutional Animal Care and Use Committee. Models of endotoxin-induced lung injury (intratracheal LPS) or lung injury by live bacteria (intratracheal PA) were employed in accordance with American Thoracic Society guidelines on animal models of experimental lung injury (56). The principal objective in these models is the physiological assessment of lung injury, and studies were powered to detect a 2-fold difference in BALF protein amount, which is a well-established primary endpoint for animal models of acute lung injury and based on our prior published results using these models of lung injury $(17,18,21,27,28,50,57)$. For intratracheal injection studies, C57BL/6J mice were anesthetized using a ketamine/xylazine mixture, and the larynx was well visualized under a fiber optic light source before endotracheal intubation with a 3/400 24-gauge plastic catheter. LPS with or without IL-3 (rIL-3) (LPS: $1.5 \mathrm{mg} / \mathrm{kg}$; rIL-3: $6 \mu \mathrm{g}$ ) were administered intratracheally (i.t.) for 18 hours, after which animals were euthanized and assayed for BALF protein amounts by Lowry assay and immunoblotting, cell count by TC20 automated cell counter, cytokine abundance by ELISA, and lung tissue H\&E staining. In another study, LPS with or without IL-3-neutralizing antibody (Cell Signaling, D6C1) (LPS: $1.5 \mathrm{mg} /$ $\mathrm{kg}$; anti-IL-3: $5 \mu \mathrm{g}$ ) was administered i.t. for 18 hours, after which animals were euthanized and assayed for BALF protein amounts by immunoblotting, cell count, cytokine abundance by ELISA, and lung tissue H\&E staining. For the lentiviral study, $1 \times 10^{7} \mathrm{PFU}$ of lentivirus encoding genes for RNFT2 or Rnft $2 \mathrm{shR}-$ NA was treated i.t. for 144 hours before the administration of LPS with or without rIL-3 (LPS: $1.5 \mathrm{mg}$ / $\mathrm{kg}$; rIL-3: $6 \mu \mathrm{g}$ in RNFT2 overexpression mice, $3 \mu \mathrm{g}$ in RNFT2 shRNA mice) for 18 hours, after which animals were euthanized and assayed for BALF protein amounts by immunoblotting, cell count, cytokine abundance by ELISA, and lung tissue H\&E staining. 
Human studies of ARDS. Deidentified human plasma samples were obtained from the University of Pittsburgh Acute Lung Injury Biospecimen Repository (IRB PR010110387). Informed consent was provided by all participants or their surrogates in accordance with the Declaration of Helsinki. Adults aged 18-90 admitted to the medical ICU in the University of Pittsburgh Medical Center Presbyterian Hospital with acute respiratory failure requiring mechanical ventilation by endotracheal tube were enrolled as patients if the onset of acute respiratory failure was associated with established risk factors for developing acute lung injury (sepsis, pneumonia, aspiration, blood transfusion, pancreatitis, or trauma) or if intubation was performed for airway protection in the setting of nonpulmonary critical illness. Enrollment took place within 48 hours of the initiation of mechanical ventilation. After enrollment, patients were retrospectively subclassified by an expert clinical panel into categories: (a) "ARDS," as defined by Berlin criteria and agreed upon by a minimum of 3 members of an expert clinical panel; (b) "at risk," as defined as Lung Injury Prediction Score score higher than 4 and the presence of a clinically identifiable risk factor for ARDS; and (c) "ventilated control," as defined by intubation and mechanical ventilation for nonpulmonary critical illness without risk factors for ARDS. We performed a preliminary sample size calculation to detect a 3-fold difference in 2 means (30 pg/ $\mathrm{mL}$ vs. $90 \mathrm{pg} / \mathrm{mL}$ ) between ventilated control and lung-injured patients with a power of $0.8, \alpha$ of 0.05 , estimated SD of 35, and sampling ratio of 0.25 ventilated control/lung-injured patients (given there were fewer control patients enrolled in our study). We chose values of 30 and $90 \mathrm{pg} / \mathrm{mL}$ based on published literature showing an IL-3 amount of more than $89.4 \mathrm{pg} / \mathrm{mL}$ is associated with poor outcomes in sepsis (2) and estimated baseline amounts from manufacturer data and published literature. The predicted sample size was 4 "control" patients and 14 "lung-injured" patients. With this, $n=4$ "ventilated control" and $n=16$ "at risk" for ARDS were randomly selected from the cohort for analysis.

Human studies of CF. Following attainment of informed consent, CF lung tissue was obtained from excess pathological tissue following lung transplantation in accordance with a protocol approved by the University of Pittsburgh IRB. Sections of lung parenchyma, $1 \mathrm{~cm}^{2}$, were frozen and stored at $-80^{\circ} \mathrm{C}$ until use. Approximately $100 \mathrm{mg}$ of lung tissue was homogenized in RIPA buffer followed by protein immunoblotting.

Data and materials availability. All data needed to evaluate the conclusions in the paper are present in the paper or the supplemental materials.

Statistics. Protein Signal densitometry was quantified by ImageJ (NIH). Comparisons of 2 groups were carried out with unpaired, 2-tailed Student's $t$ test. Comparisons of more than 2 groups were tested with 1-way ANOVA with Tukey's or Dunnett's post hoc test for multiple comparisons as noted. Nonlinear regression plots were tested with $F$ test. All tests used $P<0.05$ as indicative of statistical significance. All statistical analyses were carried out using the GraphPad Prism 8.0 program.

Study approval. C57BL/6J mice were purchased from The Jackson Laboratories. All procedures were approved by the University of Pittsburgh Institutional Animal Care and Use Committee. For ARDS human studies, deidentified human plasma samples were obtained from the University of Pittsburgh Acute Lung Injury Biospecimen Repository (IRB PR010110387). Informed consent was provided by all participants or their surrogates in accordance with the Declaration of Helsinki. Following attainment of informed consent, CF lung tissue was obtained from excess pathological tissue following lung transplantation in accordance with a protocol approved by the University of Pittsburgh IRB.

\section{Author contributions}

$\mathrm{BBC}, \mathrm{YL}$, and MJJ designed the study and analyzed the data; YT, TBL, JE, and BBC wrote the manuscript; YT, TBL, JE, KCL, YC, and JDL performed all in vitro experiments and animal experiments; and JE, MMM, YZ, IDP, JFM, and BJM designed and executed the human studies. BBC directed the study.

\section{Acknowledgments}

We wish to offer a special acknowledgment to the patients and their families who were enrolled in the University of Pittsburgh Acute Lung Injury Biospecimen Repository. This work was supported by the National Institutes of Health R35 grant HL139860 (to BBC), P50 grant P50AR060780 (to BBC), R01 grant HL133184 (to JM and BBC), China National Natural Science Foundation grant 81900073 (to YT), F31 grant HL143843 (to TBL), K08 grant HL144820 (to JE), American Heart Association Scientist Development Grant 16SDG27650008 (to YL), R01 grant HL14277 (to YL), R01 grant DK119627 (to MJJ and YL), CFF (Research Development Program to the University of Pittsburgh) and P30 DK072506 (to MMM), and P01 grant HL114453 (to BJM, YZ, and BBC). 
Address correspondence to: Bill B. Chen or Yuan Liu, University of Pittsburgh, Aging Institute, Department of Medicine, 558 Bridgeside Point 1, 100 Technology Drive, Pittsburgh, Pennsylvania 15219, USA. Phone: 412.624.8900; Email: chenb@upmc.edu (BBC); yul119@pitt.edu (YL).

1. Broughton SE, et al. The GM-CSF/IL-3/IL-5 cytokine receptor family: from ligand recognition to initiation of signaling. Immunol Rev. 2012;250(1):277-302.

2. Weber GF, et al. Interleukin-3 amplifies acute inflammation and is a potential therapeutic target in sepsis. Science. 2015;347(6227):1260-1265.

3. Takai S, Yamada K, Hirayama N, Miyajima A, Taniyama T. Mapping of the human gene encoding the mutual signal-transducing subunit (beta-chain) of granulocyte-macrophage colony-stimulating factor (GM-CSF), interleukin-3 (IL-3), and interleukin-5 (IL-5) receptor complexes to chromosome 22q13.1. Hum Genet. 1994;93(2):198-200.

4. Hu J, et al. The inhibitor of interleukin-3 receptor protects against sepsis in a rat model of cecal ligation and puncture. Mol Immunol. 2019;109:71-80.

5. Bentzer P, Fjell C, Walley KR, Boyd J, Russell JA. Plasma cytokine levels predict response to corticosteroids in septic shock. Intensive Care Med. 2016;42(12):1970-1979.

6. Broughton SE, et al. The $\beta c$ receptor family - structural insights and their functional implications. Cytokine. 2015;74(2):247-258.

7. Hercus TR, et al. Role of the $\beta$ Common ( $\beta c$ ) Family of Cytokines in Health and Disease. Cold Spring Harb Perspect Biol. 2018;10(6):a028514

8. Meads MB, Li ZW, Dalton WS. A novel TNF receptor-associated factor 6 binding domain mediates NF-kappa B signaling by the common cytokine receptor beta subunit. J Immunol. 2010;185(3):1606-1615.

9. Borriello F, Galdiero MR, Varricchi G, Loffredo S, Spadaro G, Marone G. Innate Immune Modulation by GM-CSF and IL-3 in Health and Disease. Int J Mol Sci. 2019;20(4):E834.

10. Rytkönen A, Holden DW. Bacterial interference of ubiquitination and deubiquitination. Cell Host Microbe. 2007;1(1):13-22.

11. Morreale FE, Walden H. Types of ubiquitin ligases. Cell. 2016;165(1):248-248.e1.

12. Metzger MB, Hristova VA, Weissman AM. HECT and RING finger families of E3 ubiquitin ligases at a glance. J Cell Sci. 2012;125(Pt 3):531-537.

13. Borden KL, Freemont PS. The RING finger domain: a recent example of a sequence-structure family. Curr Opin Struct Biol. 1996;6(3):395-401.

14. Freemont PS, Hanson IM, Trowsdale J. A novel cysteine-rich sequence motif. Cell. 1991;64(3):483-484.

15. Lovering R, et al. Identification and preliminary characterization of a protein motif related to the zinc finger. Proc Natl Acad Sci US A. 1993;90(6):2112-2116.

16. Jin J, et al. Proinflammatory TLR signalling is regulated by a TRAF2-dependent proteolysis mechanism in macrophages. Nat Commun. 2015;6:5930.

17. McKelvey AC, et al. RING finger E3 ligase PPP1R11 regulates TLR2 signaling and innate immunity. Elife. 2016;5:e18496.

18. Lear T, et al. Ubiquitin E3 ligase FIEL1 regulates fibrotic lung injury through SUMO-E3 ligase PIAS4. J Exp Med. 2016;213(6):1029-1046.

19. $\mathrm{Hu} \mathrm{H}$, et al. Otud7b facilitates $\mathrm{T}$ cell activation and inflammatory responses by regulating Zap70 ubiquitination. J Exp Med. 2016;213(3):399-414.

20. Chang M, Jin W, Sun SC. Peli1 facilitates TRIF-dependent Toll-like receptor signaling and proinflammatory cytokine production. Nat Immunol. 2009;10(10):1089-1095.

21. Coon TA, et al. The proinflammatory role of HECTD2 in innate immunity and experimental lung injury. Sci Transl Med. 2015;7(295):295ra109.

22. Jia Y, et al. Lamin B1 loss promotes lung cancer development and metastasis by epigenetic derepression of RET. J Exp Med. 2019;216(6):1377-1395.

23. Komander D, Rape M. The ubiquitin code. Annu Rev Biochem. 2012;81:203-229.

24. Hospenthal MK, Mevissen TET, Komander D. Deubiquitinase-based analysis of ubiquitin chain architecture using Ubiquitin Chain Restriction (UbiCRest). Nat Protoc. 2015;10(2):349-361.

25. Ravid T, Hochstrasser M. Diversity of degradation signals in the ubiquitin-proteasome system. Nat Rev Mol Cell Biol. 2008;9(9):679-690.

26. Evankovich J, et al. Receptor for advanced glycation end products is targeted by FBXO10 for ubiquitination and degradation. FASEB J. 2017;31(9):3894-3903.

27. Chen BB, et al. E3 ligase subunit Fbxo15 and PINK1 kinase regulate cardiolipin synthase 1 stability and mitochondrial function in pneumonia. Cell Rep. 2014;7(2):476-487.

28. Chen BB, et al. A combinatorial $\mathrm{F}$ box protein directed pathway controls TRAF adaptor stability to regulate inflammation. Nat Immunol. 2013;14(5):470-479.

29. Min J, et al. Integrated biosensor for rapid and point-of-care sepsis diagnosis. ACS Nano. 2018;12(4):3378-3384.

30. Calfee CS, et al. Subphenotypes in acute respiratory distress syndrome: latent class analysis of data from two randomised controlled trials. Lancet Respir Med. 2014;2(8):611-620.

31. Delucchi K, et al. Stability of ARDS subphenotypes over time in two randomised controlled trials. Thorax. 2018;73(5):439-445.

32. Srivastava RK, et al. IL-3 attenuates collagen-induced arthritis by modulating the development of Foxp3+ regulatory T cells. $J$ Immunol. 2011;186(4):2262-2272.

33. Renner K, et al. IL-3 promotes the development of experimental autoimmune encephalitis. JCI Insight. 2016;1(16):e87157.

34. Renner K, et al. IL-3 contributes to development of lupus nephritis in MRL/lpr mice. Kidney Int. 2015;88(5):1088-1098.

35. Middelburg RA, van der Bom JG. Transfusion-related acute lung injury not a two-hit, but a multicausal model. Transfusion. 
2015;55(5):953-960

36. Birukova AA, Tian Y, Meliton A, Leff A, Wu T, Birukov KG. Stimulation of Rho signaling by pathologic mechanical stretch is a "second hit" to Rho-independent lung injury induced by IL-6. Am J Physiol Lung Cell Mol Physiol. 2012;302(9):L965-L975.

37. Walsh MC, Lee J, Choi Y. Tumor necrosis factor receptor- associated factor 6 (TRAF6) regulation of development, function, and homeostasis of the immune system. Immunol Rev. 2015;266(1):72-92.

38. Inoue Ji, et al. Tumor necrosis factor receptor-associated factor (TRAF) family: adapter proteins that mediate cytokine signaling. Exp Cell Res. 2000;254(1):14-24.

39. Häcker H, et al. Specificity in Toll-like receptor signalling through distinct effector functions of TRAF3 and TRAF6. Nature 2006;439(7073):204-207.

40. Cohen P. The TLR and IL-1 signalling network at a glance. J Cell Sci. 2014;127(Pt 11):2383-2390.

41. Kawasaki T, Kawai T. Toll-like receptor signaling pathways. Front Immunol. 2014;5:461.

42. Hu H, Sun SC. Ubiquitin signaling in immune responses. Cell Res. 2016;26(4):457-483.

43. Yang XD, Sun SC. Targeting signaling factors for degradation, an emerging mechanism for TRAF functions. Immunol Rev. 2015;266(1):56-71.

44. Fang R, et al. MAVS activates TBK1 and IKKe through TRAFs in NEMO dependent and independent manner. PLoS Pathog. 2017;13(11):e1006720

45. Shi M, et al. TRIM30 alpha negatively regulates TLR-mediated NF-kappa B activation by targeting TAB2 and TAB3 for degradation. Nat Immunol. 2008;9(4):369-377.

46. Chousterman BG, Swirski FK. Innate response activator B cells: origins and functions. Int Immunol. 2015;27(10):537-541.

47. Du Y, et al. Lung Gene Expression Analysis (LGEA): an integrative web portal for comprehensive gene expression data analysis in lung development. Thorax. 2017;72(5):481-484.

48. Du Y, Guo M, Whitsett JA, Xu Y. 'LungGENS': a web-based tool for mapping single-cell gene expression in the developing lung. Thorax. 2015;70(11):1092-1094.

49. Montoro DT, et al. A revised airway epithelial hierarchy includes CFTR-expressing ionocytes. Nature. 2018;560(7718):319-324.

50. Zhao J, et al. F-box protein FBXL19-mediated ubiquitination and degradation of the receptor for IL-33 limits pulmonary inflammation. Nat Immunol. 2012;13(7):651-658.

51. Liu Y, et al. The proapoptotic F-box protein Fbxl7 regulates mitochondrial function by mediating the ubiquitylation and proteasomal degradation of survivin. J Biol Chem. 2015;290(19):11843-11852.

52. Han S, et al. Lipopolysaccharide primes the NALP3 inflammasome by inhibiting its ubiquitination and degradation mediated by the SCFFBXL2 E3 ligase. J Biol Chem. 2015;290(29):18124-18133.

53. Townsend MH, Shrestha G, Robison RA, O'Neill KL. The expansion of targetable biomarkers for CAR T cell therapy. J Exp Clin Cancer Res. 2018;37(1):163.

54. Testa U, Pelosi E, Frankel A. CD 123 is a membrane biomarker and a therapeutic target in hematologic malignancies. Biomark Res. 2014;2(1):4.

55. Lim KL, et al. Parkin mediates nonclassical, proteasomal-independent ubiquitination of synphilin-1: implications for Lewy body formation. J Neurosci. 2005;25(8):2002-2009.

56. Matute-Bello G, et al. An official American Thoracic Society workshop report: features and measurements of experimental acute lung injury in animals. Am J Respir Cell Mol Biol. 2011;44(5):725-738.

57. Zou C, et al. Mortality factor 4 like 1 protein mediates epithelial cell death in a mouse model of pneumonia. Sci Transl Med. 2015;7(311):311ra171. 